\title{
SFT non-locality in cosmology: solutions, perturbations and observational evidences
}

\author{
Alexey S. Koshelev ${ }^{1}$ \\ Theoretische Natuurkunde, Vrije Universiteit Brussel and The International Solvay Institutes, Pleinlaan 2, \\ B-1050 Brussels, Belgium
}

\begin{abstract}
In this note cosmological models coming out of the String Field Theory (SFT) in application to the Dark Energy are reviewed. A way of constructing solutions in the case of linear models is outlined, cosmological perturbations and observational evidences of such models are explored. We explicitly demonstrate the stability of the system at the linear order in the most typical configuration.
\end{abstract}

Keywords: String Field Theory, Rolling tachyon, Cosmological perturbations, Dark Energy

PACS: 11.25.-w,11.25.Sq,98.80.-k,98.80.Jk

\section{INTRODUCTION}

In this note we briefly review the recent activity on a new class of cosmological models based on the string field theory (SFT) (for details see reviews [1]) and the $p$-adic string theory [2] focusing on their applications to the present Dark Energy phenomenon. The reader is referred to $[3,4,5,6]$ and refs. therein for more detailed analysis on the subject. It is known that the SFT and the $p$-adic string theory are UV-complete ones. Thus one can expect that resulting (effective) models should be free of pathologies. Furthermore, models originating from the SFT exhibit one general non-standard property, namely, they have terms with infinitely many derivatives, i.e. non-local terms. The higher derivative terms usually produce the well known Ostrogradski instability [7]. However the Ostrogradski result is related to higher than two but finite number of derivatives. In the case of infinitely many derivatives it is possible that instabilities do not appear.

Contemporary cosmological observational data $[8,9]$ strongly support that the present Universe exhibits an accelerated expansion providing thereby an evidence for a dominating DE component [10]. Recent results of WMAP [9] together with the data on Ia supernovae give the following bounds for the DE state parameter $w_{\mathrm{DE}}=-1.02_{-0.16}^{+0.14}$. Note that the present cosmological observations do not exclude an evolving DE state parameter $w$. Non-local models of the type one has from the SFT may have effective phantom behavior and are of interest for the present cosmology. To construct a stable model with $w<-1$ one should construct the effective theory with the Null Energy Condition (NEC) violation from the fundamental theory, which is stable and admits quantization. This is a hint towards the SFT inspired cosmological models.

In this note we shall demonstrate how an effective phantom behavior may appear, shall consider an indicative example along with cosmological perturbations arguing that the phantom phase in these models does not produce growing perturbations and shall outline a possible signature of such non-local models.

\section{THE MODEL}

We are interested in non-local models arising from SFT in the far asymptotic regime for large times. The starting point is the Lagrangian

$$
S=\int d^{4} x \sqrt{-g}\left(\frac{R}{16 \pi G_{N}}+\frac{1}{g_{o}^{2}}\left(-\frac{1}{2} \partial_{\mu} T \partial^{\mu} T+\frac{1}{2 \alpha^{\prime}} T^{2}-\frac{1}{\alpha^{\prime}} v(\bar{T})\right)-\Lambda^{\prime}\right) .
$$

\footnotetext{
${ }^{1}$ Postdoctoral researcher of FWO-Vlaanderen, e-mail: alexey .koshelev@vub.ac . be.
} 
where we manifestly indicate four dimensions despite the stringy origin. We work in $1+3$ dimensions with the signature $(-,+,+,+)$, the coordinates are denoted by Greek indices $\mu, v, \ldots$ running from 0 to 3 . Spatial indexes are $a, b, \ldots$ and they run from 1 to 3 . Such four-dimensional action is motivated by the string field theory and details can be found in [4]. $G_{N}$ is the Newtonian constant: $8 \pi G_{N}=1 / M_{P}^{2}$, where $M_{P}$ is the Planck mass, $\alpha^{\prime}$ is the string length squared (we do not assume $\alpha^{\prime}=1 / M_{P}^{2}$ ), $g_{o}$ is the open string coupling constant. $g_{\mu v}$ is the metric tensor, $R$ is the scalar curvature, $\Lambda^{\prime}$ is a constant, $\bar{T}=\mathscr{G}\left(\alpha^{\prime} \square\right) T$ with

$$
\square=D^{\mu} \partial_{\mu}=\frac{1}{\sqrt{-g}} \partial_{\mu} \sqrt{-g} g^{\mu v} \partial_{v}
$$

and $D_{\mu}$ being a covariant derivative, $T$ is a scalar field primarily associated with the open string tachyon. The function $\mathscr{G}\left(\alpha^{\prime} \square\right)$ may be not a polynomial manifestly producing thereby the non-locality. In SFT one has $\mathscr{G}\left(\alpha^{\prime} \square\right)=e^{-\frac{\beta}{2} \alpha^{\prime} \square}$ but we keep this function general. $\beta$ is a parameter determined exclusively by the conformal field theory of the string. Here we see the very important feature: we may have non-locality in the action. We stress here that appearance of non-localities is general feature of the SFT based models and exactly the one we are going to explore ${ }^{2}$. Fields are dimensionless while $\left[g_{o}\right]=$ length. $v(\bar{T})$ is an open string tachyon self-interaction. It does not have a quadratic in $T$ term. Factor $1 / \alpha^{\prime}$ in front of the tachyon potential looks unusual and can be easily removed by a rescaling of fields. For our purposes it is convenient keeping all the fields dimensionless.

Redefining the tachyon field $T_{b}=\bar{T}$ and introducing dimensionless coordinates $\bar{x}_{\mu}=x_{\mu} / \sqrt{\alpha^{\prime}}$ the above action can be rewritten as (where we omit bars for simplicity and put hereafter $\alpha^{\prime}=1$ )

$$
S=\int d^{4} x \sqrt{-g}\left(\frac{R}{16 \pi G_{N}}+\frac{1}{g_{o}^{2}}\left(-\frac{1}{2} \partial_{\mu} \tilde{T}_{b} \partial^{\mu} \tilde{T}_{b}+\frac{1}{2} \tilde{T}_{b}^{2}-v\left(T_{b}\right)\right)-\Lambda^{\prime}\right) .
$$

Let us emphasize that the potential of the field $T_{b}$ is $V=-\frac{1}{2} T_{b}^{2}+v\left(T_{b}\right)$. Given an extremum of the potential $V$ one can linearize the theory around it using $T_{b}=T_{0}-\tau$. This gives $V=-\frac{1}{2} \tau^{2}+\frac{v\left(T_{0}\right)^{\prime \prime}}{2} \tau^{2}+V\left(T_{0}\right)$. Action (3) being linearized around the extremum of the potential of the field $T_{b}$ can be written as

$$
S=\int d^{4} x \sqrt{-g}\left(\frac{R}{16 \pi G_{N}}+\frac{1}{2 g_{o}^{2}} \tau \mathscr{F}(\square) \tau-\Lambda\right),
$$

where

$$
\mathscr{F}(\square)=(\square+1) \mathscr{G}^{-2}(\square)-m^{2} .
$$

Here $m^{2} \equiv v\left(T_{0}\right)^{\prime \prime}$ and $\Lambda$ accounts $\frac{V\left(T_{0}\right)}{g_{o}^{2}}$. From SFT one has

$$
\mathscr{F}_{\mathrm{SFT}}(\square)=(\square+1) e^{\beta \square}-m^{2}
$$

$\mathscr{F}$ is in fact the inverse propagator and it is natural to expect $\beta<0$ in the SFT case corresponding to a convergent propagator at large momenta. In this SFT example we can learn a very important thing. Assume $m=0$. Then $\mathscr{F}_{\mathrm{SFT}}=(\square+1) e^{\beta \square}$ and the non-locality does not show up at all. Indeed, the poles of the propagator do not feel the exponent. Other way of thinking is that the exponential factor can be hidden by a field redefinition. The situation is dramatically different for $m \neq 0$. The propagator $1 / \mathscr{F}_{\mathrm{SFT}}$ has infinitely many poles. This is the manifestation of the non-locality. Also we stress that physics would be totally different for full function $\mathscr{F}$ and its truncated series expansion because the pole structure may get modified considerably.

We see that the most important role is played by the spectrum of the theory which is determined by the equation

$$
\mathscr{F}(J)=(J+1) \mathscr{G}^{-2}(J)-m^{2}=0 .
$$

We name it characteristic equation. It can be an algebraic or a transcendental one. Thus until examples are considered we shall keep $\mathscr{F}(\square)$ general. The only assumption is that all roots are simple. Also the analyticity of the function $\mathscr{F}$ on the complex plane is important so that one can represent $\mathscr{F}$ by the convergent series expansion:

$$
\mathscr{F}=\sum_{n=0}^{\infty} f_{n} \square^{n} \text { and } f_{n} \in \mathbb{R} .
$$

\footnotetext{
${ }^{2}$ Appearance of higher derivatives is not unique for this theory. Non-commutative theories, for instance, also have higher derivative, but these non-local structures are very different.
} 
Reality of coefficients is required by the hermiticity of the Lagrangian. Strictly speaking even the analyticity requirement can be weakened but in this case one has to be careful with poles and consider only the appropriate domains of the argument were the series expansion does converge.

Equations of motion are

$$
\begin{aligned}
G_{\mu \nu}=\frac{8 \pi G_{N}}{g_{o}^{2}} T_{\mu \nu}= & 4 \pi G \sum_{n=1}^{\infty} f_{n} \sum_{l=0}^{n-1}\left(\partial_{\mu} \square^{l} \tau \partial_{\nu} \square^{n-1-l} \tau+\partial_{\nu} \square^{l} \tau \partial_{\mu} \square^{n-1-l} \tau-\right. \\
& \left.-g_{\mu \nu}\left(g^{\rho \sigma} \partial_{\rho} \square^{l} \tau \partial_{\sigma} \square^{n-1-l} \tau+\square^{l} \tau \square^{n-l} \tau\right)\right)-8 \pi G_{N} g_{\mu \nu} \Lambda,
\end{aligned}
$$

where $G_{\mu \nu}$ is the Einstein tensor, $T_{\mu \nu}$ is the energy-momentum (stress) tensor and $G \equiv G_{N} / g_{o}^{2}$ is a dimensionless analog of the Newtonian constant. It is easy to check that the Bianchi identity is satisfied on-shell and for $\mathscr{F}=f_{1} \square+f_{0}$ the usual energy-momentum tensor for the massive scalar field is reproduced. Note that equation (10) is an independent equation consistent with system (9) due to the Bianchi identity.

Classical solutions to equations (9) and (10) were studied and analyzed in the literature. The cornerstone in this analysis is the fact that action (4) is fully equivalent to the following action with many free local scalar fields

$$
S_{\text {local }}=\int d^{4} x \sqrt{-g}\left(\frac{R}{16 \pi G_{N}}-\frac{1}{g_{o}^{2}} \sum_{i} \frac{\mathscr{F}^{\prime}\left(J_{i}\right)}{2}\left(g^{\mu v} \partial_{\mu} \tau_{i} \partial_{v} \tau_{i}+J_{i} \tau_{i}^{2}\right)-\Lambda\right)
$$

Here there are as many scalar fields as many roots the characteristic equation (7) has.

To see the equivalence first we write down the equations of motion for the latter local action which are

$$
\begin{aligned}
G_{\mu \nu}=\frac{8 \pi G_{N}}{g_{o}^{2}} T_{\mu \nu} & =\frac{8 \pi G_{N}}{g_{o}^{2}} \sum_{i} \mathscr{F}^{\prime}\left(J_{i}\right)\left(\partial_{\mu} \tau_{i} \partial_{\nu} \tau_{i}-\frac{1}{2} g_{\mu \nu}\left(g^{\rho \sigma} \partial_{\rho} \tau_{i} \partial_{\sigma} \tau_{i}+J_{i} \tau_{i}^{2}\right)\right)- \\
& -8 \pi G_{N} g_{\mu \nu} \Lambda
\end{aligned}
$$

and

$$
\square \tau_{i}=J_{i} \tau_{i}, \text { for all } i
$$

Note that $J_{i}$ is a root of characteristic equation (7). Therefore any $\tau_{i}$ solves equation (10) just because

$$
\mathscr{F}(\square) \tau_{i}=\mathscr{F}\left(J_{i}\right) \tau_{i}=0, \text { for all } i
$$

As a consequence of linearity of equation (10) its solution is a linear combination $\sum_{i} \tau_{i}$. Any arbitrary coefficients in this summation can be accounted by the integration constants in each $\tau_{i}$. Substituting $\tau=\sum_{i} \tau_{i}$ in (9) one can readily find that equation (9) is transformed into equation (12). To complete the proof of the equivalence statement one has to show that $\tau=\sum_{i} \tau_{i}$ is a general solution to equation (10). This was done for a large class of functions $\mathscr{F}(\square)$.

It is important to understand at this point that roots $J_{i}$ as well as coefficients $\mathscr{F}^{\prime}\left(J_{i}\right)$ can be complex. This should not spoil the theory just because all the local scalar fields are just mathematical functions. They do not have physical meaning. What is important is that the original field $\tau$ must be real since it represents a physical excitation. The latter is easy to achieve. Namely, the roots $J_{i}$ are either real or complex conjugate. Complex conjugate $J_{i}$ would yield complex conjugate (up to an overall constant factor) solutions for $\tau_{i}$. Thus, it is a matter of choice of the integration constants to make the linear combination of all $\tau_{i}$ real. Moreover, coefficients $\mathscr{F}^{\prime}\left(J_{i}\right)$ can be absorbed in the scalar fields by the field rescaling. We do not do this just because real $\mathscr{F}^{\prime}\left(J_{i}\right)$ would indicate whether the corresponding field is a phantom or not. Also we note that the above proven equivalence is true for arbitrary metric.

We continue with local action (11). Moreover, from now on we specialize to the spatially flat Friedmann-RobertsonWalker (FRW) Universe. The metric is of the form

$$
d s^{2}=-d t^{2}+a^{2}(t)\left(d x_{1}^{2}+d x_{2}^{2}+d x_{3}^{2}\right)
$$

where $a(t)$ is the scale factor, $t$ is the cosmic time. The Hubble parameter is as usual $H=\dot{a} / a$ and the dot hereafter in this paper denotes a derivative with respect to the cosmic time $t$. Background solutions for $\tau$ are taken to be spacehomogeneous as well. Equations of motion become much simpler.

$$
3 H^{2}=4 \pi G \sum_{i} \mathscr{F}_{,}^{\prime}\left(M_{i}\right)\left(\dot{\tau}_{i}^{2}+M_{i} \tau_{i}^{2}\right)+8 \pi G_{N} \Lambda, \dot{H}=-4 \pi G \sum_{i} \mathscr{F}_{, M}^{\prime}\left(M_{i}\right) \dot{\tau}_{i}^{2} .
$$


and

$$
\ddot{\tau}_{i}+3 H \dot{\tau}_{i}+J_{i} \tau_{i}=0, \text { for all } i
$$

It was proven in [6] that cosmological perturbations are also equivalent in the free theory with one non-local scalar field (4) and in the corresponding local theory (11) with many scalar field. In the next Section we shall consider the most important example with two complex conjugate roots $J_{i}$.

Obviously if we have two consequent real roots $J_{1}$ and $J_{2}$ then $\mathscr{F}^{\prime}\left(J_{1}\right)$ and $\mathscr{F}^{\prime}\left(J_{2}\right)$ are real and would have different signs. This is one the origin of the phantom like behavior in such models. Such phantom behavior, however, is not distinguishable from the already known examples. Complex roots $J_{i}$ in turn also produce the phantom-like behavior but this one is very different from the previous case. Indeed, suppose we have only two complex conjugate roots $J_{1}$ and $J_{2}=J_{1}^{*}$ in action (11). Then one can easily pass to the real components of the fields. However, such real fields will be quadratically coupled. Thus, the intuition based on the signs of kinetic terms and mass terms will be broken. New behavior is reflected in the fact that solutions are classically stable and perturbations do not grow even though the phantom divide can be crossed.

\section{EXAMPLE OF BACKGROUND SOLUTION}

The reduction to a one-field model is possible but practically is trivial. Nothing new arises because the structure coming from the SFT is not felt. One pair of complex conjugate roots $J_{1}=J$ and $J_{2}=J^{*}$ is a new situation worth the attention.

It is obvious that if a complex number $J$ is a root of $\mathscr{F}$, then $J^{*}$ is a root of $\mathscr{F}$ as well. The system of Friedmann equations becomes:

$$
\begin{aligned}
3 H^{2} & =4 \pi G\left[\mathscr{F}^{\prime}(J)\left(\dot{\tau}_{1}^{2}+J \tau_{1}^{2}\right)+\mathscr{F}^{\prime}\left(J^{*}\right)\left(\dot{\tau}_{2}^{2}+J^{*} \tau_{2}{ }^{2}\right)\right]+8 \pi G_{N} \Lambda, \\
\dot{H} & =-4 \pi G\left[\mathscr{F}^{\prime}(J) \dot{\tau}_{1}{ }^{2}+\mathscr{F}^{\prime}\left(J^{*}\right) \dot{\tau}_{2}{ }^{2}\right] .
\end{aligned}
$$

This is a really new configuration much less explored in the past. The distinguishing feature is the complex coefficients. Because of this solutions for the scalar fields also expected to be complex. However, as it was already stressed these fields are not physical. What is physical is their linear combination and only the latter must be real. This is easy to achieve adjusting the integration constants, say, $\tau_{1}=\tau_{2}^{*}$. What is interesting (but not surprising, though) one cannot have non-interacting fields passing to the real components. Precisely, fields will be quadratically coupled in the Lagrangian. This means that the usual intuition about field properties (like signs of coefficients in front the kinetic term or the mass term) may not work. In addition to the latter system of equations we can use the equations of motion for the scalar fields which are

$$
\ddot{\tau}_{1}+3 H \dot{\tau}_{1}+J \tau_{1}=0, \quad \ddot{\tau}_{2}+3 H \dot{\tau}_{2}+J^{*} \tau_{2}=0
$$

Equations (17) and (18) describe the late time evolution of the model with Lagrangian (3). This model possess a solution with the scalar field tending to the minimum of the potential (i.e. $\tau \rightarrow 0$ ) and the Hubble parameter going to the constant. Such solution was constructed numerically and was proven to be a solution [11]. Also the asymptotic form of this solution was derived in [4]. The idea is to compute a solution to (18) using the constant $H=H_{0}$ and then compute the correction to $H$ using (17). Then the procedure can be iterated to give higher and higher corrections. It was proven in [4] such iterations do converge.

Solution to (18) with constant $H=H_{0}$ is obviously

$$
\tau_{1}=\tau_{1+} e^{\alpha_{+} t}+\tau_{1-} e^{\alpha_{-} t}, \tau_{2}=\tau_{2+} e^{\alpha_{+}^{*} t}+\tau_{2-} e^{\alpha_{-}^{*} t}
$$

where $\alpha_{ \pm}=\frac{3 H_{0}}{2}\left(-1 \pm \sqrt{1-\frac{4 J}{9 H_{0}^{2}}}\right)$. Consider $\tau_{1}$. Only one term in the solution converges when $t \rightarrow \infty$ in general (if both converge we select the slowest one). Let's assume it is the first one proportional to $\tau_{1+}$ constant. Then in order to pick the (slowest) convergent solution we put $\tau_{1-}=0$. The similar term with $\tau_{2+}$ will converge in $\tau_{2}{ }^{3}$. To assure the initial field $\tau$ is real we have to impose $\tau_{2+}=\tau_{1+}^{*}$. Here we define $\tau_{1+} \equiv \tau_{0}$ and $\alpha_{+} \equiv \alpha$.

\footnotetext{
${ }^{3}$ Here we adopt the rule $\sqrt{z^{*}}=\sqrt{z}$ meaning that the phase of the complex number runs in the interval $[-\pi, \pi)$ and for $z=r e^{i \varphi}$ the square root is $|\sqrt{r}| e^{i \varphi / 2}$.
} 
The first correction to the constant Hubble parameter in case only decaying modes in $\tau$ are present is

$$
H=H_{0}+h=H_{0}+h_{0}\left(\tau_{1}^{2}+\tau_{1}^{* 2}\right)
$$

Constant $h_{0}$ is not independent and is related with $\tau_{0}$. We note that $h$ is of order $\tau^{2}$. The last expression is a good approximation for $H$ in the asymptotic regime when $h \ll H_{0}$.

For the energy and the pressure we find

$$
\begin{aligned}
& \rho=\frac{1}{2}\left[\mathscr{F}^{\prime}(J) \tau_{1}^{2}\left(\alpha^{2}+J\right)+\mathscr{F}^{\prime}\left(J^{*}\right) \tau_{1}^{* 2}\left(\alpha^{* 2}+J^{*}\right)\right]+g_{o}^{2} \Lambda, \\
& p=\frac{1}{2}\left[\mathscr{F}^{\prime}(J) \tau_{1}^{2}\left(\alpha^{2}-J\right)+\mathscr{F}^{\prime}\left(J^{*}\right) \tau_{1}^{* 2}\left(\alpha^{* 2}-J^{*}\right)\right]-g_{o}^{2} \Lambda
\end{aligned}
$$

and consequently for the state parameter and the speed of sound

$$
\begin{aligned}
w & \approx-1-\frac{\mathscr{F}^{\prime}(J) J \tau_{1}^{2}+\mathscr{F}^{\prime}\left(J^{*}\right) J^{*} \tau_{1}^{* 2}}{g_{o}^{2} \Lambda}, \\
c_{s}^{2} & =\frac{\mathscr{F}^{\prime}(J) \alpha \tau_{1}^{2}\left(\alpha^{2}-J\right)+\mathscr{F}^{\prime}\left(J^{*}\right) \alpha^{*} \tau_{1}^{* 2}\left(\alpha^{* 2}-J^{*}\right)}{\mathscr{F}^{\prime}(J) \alpha \tau_{1}^{2}\left(\alpha^{2}+J\right)+\mathscr{F}^{\prime}\left(J^{*}\right) \alpha^{*} \tau_{1}^{* 2}\left(\alpha^{* 2}+J^{*}\right)} .
\end{aligned}
$$

Further one can find the scale factor to be

$$
a=a_{0} \exp \left(H_{0} t+\frac{h_{0}}{2}\left(\frac{\tau_{1}^{2}}{\alpha}+\frac{\tau_{1}^{* 2}}{\alpha^{*}}\right)\right) .
$$

It is manifest that the phantom divide is crossed thus periodically triggering the phantom phase of the evolution. The next problem is the behavior of the cosmological perturbations. The main question: do they grow as one would expect in the ordinary phantom model or not?

\section{COSMOLOGICAL PERTURBATIONS IN THE NEIGHBORHOOD OF THE SOLUTION WITH COMPLEX MASSES}

Configurations with a single scalar field were widely studied and those appearing in the non-local models do not have any distinguished properties. Roughly speaking configurations with many scalar fields were explored as well but we have here new models featuring complex masses and complex coefficients in front of the kinetic terms. As it was stressed above there is no problem with this for the physics of our models while properties of such models, in particular the cosmological perturbations with such scalar fields were not studied in depth. Thus we focus on perturbations in the configuration with complex roots $J$. The simplest case is one pair of complex conjugate roots where the background quantities were derived in previous Section.

To find out the energy density perturbations we must use a system of equations on $\varepsilon$ which is the invariant energy density perturbation and on $\zeta$ which is the gauge invariant scalar field perturbation (see, for instance, [12, 13, 14]). The system is

$$
\begin{aligned}
& (\rho+p)\left(\ddot{\zeta}+\left(3 H_{0}+\alpha+\alpha^{*}\right) \dot{\zeta}+\left(-3 \dot{H}+\frac{k^{2}}{a_{0}^{2}} e^{-2 H_{0} t}\right) \zeta\right)= \\
= & \left(\frac{J}{\alpha}-\frac{J^{*}}{\alpha^{*}}\right)\left(\left[\mathscr{F}^{\prime}\left(J^{*}\right) \alpha^{* 2} \tau_{1}^{* 2}-\mathscr{F}^{\prime}(J) \alpha^{2} \tau_{1}^{2}\right] \dot{\zeta}+2 g_{o}^{2} \Lambda \varepsilon\right) \\
& (\rho+p)\left(\ddot{\varepsilon}+\dot{\varepsilon} H_{0}\left(8+3 c_{s}^{2}\right)+\varepsilon\left(15 H_{0}^{2}+9 H_{0}^{2} c_{s}^{2}+\frac{k^{2}}{a_{0}^{2}} e^{-2 H_{0} t}\right)\right)= \\
= & \frac{2 k^{2} \mathscr{F}^{\prime}(J) \mathscr{F}^{\prime}\left(J^{*}\right) \alpha^{2} \alpha^{* 2} \tau_{0}^{2} \tau_{0}^{* 2}}{a_{0}^{2} g_{o}^{2} \Lambda}\left(\frac{J}{\alpha}-\frac{J^{*}}{\alpha^{*}}\right) e^{2\left(-H_{0}+\alpha+\alpha^{*}\right) t} \zeta .
\end{aligned}
$$


where we should use

$$
\begin{aligned}
\dot{H} & =2 h_{0}\left(\tau_{1}^{2} \alpha+\tau_{1}^{* 2} \alpha^{*}\right) \\
\rho+p & =\mathscr{F}^{\prime}(J) \tau_{1}^{2} \alpha^{2}+\mathscr{F}^{\prime}\left(J^{*}\right) \tau_{1}^{* 2} \alpha^{* 2} \\
c_{s}{ }^{2} & =\frac{\mathscr{F}^{\prime}(J) \alpha \tau_{1}^{2}\left(\alpha^{2}-J\right)+\mathscr{F}^{\prime}\left(J^{*}\right) \alpha^{*} \tau_{1}^{* 2}\left(\alpha^{* 2}-J^{*}\right)}{\mathscr{F}^{\prime}(J) \alpha \tau_{1}^{2}\left(\alpha^{2}+J\right)+\mathscr{F}^{\prime}\left(J^{*}\right) \alpha^{*} \tau_{1}^{* 2}\left(\alpha^{* 2}+J^{*}\right)}
\end{aligned}
$$

Here $H$ is given by (20), $a$ is given by (23), energy and pressure are given by (21) and $w$ and $c_{s}^{2}$ follow from (22). Note that $\varepsilon$ is a real function and $\zeta$ is an imaginary function of time only. The latter system of equations is ready to be solved numerically but in order to get some insight in what is going on it is instructive to make some assumptions about the value $J$. This makes some analytic progress possible.

We recall the SFT origin of the model. Practically this means that values of $J$ are determined with the string scales while $H_{0}$ is expected to be much smaller. Therefore, it is natural to assume that $|\sqrt{J}| \gg H_{0}$. This implies

$$
\begin{gathered}
\alpha \approx i \sqrt{J}, \alpha^{2} \approx-J-i 3 H_{0} \sqrt{J} \\
\alpha^{*} \approx-i \sqrt{J^{*}}, \alpha^{* 2} \approx-J^{*}+i 3 H_{0} \sqrt{J^{*}}
\end{gathered}
$$

under this assumption the system $(24,25)$ becomes

$$
\begin{gathered}
(\rho+p) \ddot{\zeta}+2 i\left(\mathscr{F}^{\prime}(J) J \tau_{1}^{2} \sqrt{J^{*}}-\mathscr{F}^{\prime}\left(J^{*}\right) J^{*} \tau_{1}^{* 2} \sqrt{J}\right) \dot{\zeta}+ \\
+\left(-i 6 h_{0}(\rho+p)\left(\sqrt{J} \tau_{1}^{2}-\sqrt{J^{*}} \tau_{1}^{* 2}\right)+(\rho+p) \frac{k^{2}}{a_{0}^{2}}\right) \zeta= \\
=-i\left(\sqrt{J}+\sqrt{J^{*}}\right) 2 g_{o}^{2} \Lambda \varepsilon \\
(\rho+p) \ddot{\varepsilon}+\dot{\varepsilon} 2 i\left(\mathscr{F}^{\prime}(J) J \sqrt{J} \tau_{1}^{2}-\mathscr{F}^{\prime}\left(J^{*}\right) J^{*} \sqrt{J^{*}} \tau_{1}^{* 2}\right)- \\
-\varepsilon\left(\left(\frac{k^{2}}{a_{0}^{2}}-6 i H_{0} \sqrt{J}\right) \mathscr{F}^{\prime}(J) J \tau_{1}^{2}+\left(\frac{k^{2}}{a_{0}^{2}}+6 i H_{0} \sqrt{J^{*}}\right) \mathscr{F}^{\prime}\left(J^{*}\right) J^{*} \tau_{1}^{* 2}\right)= \\
=-i \frac{2 k^{2} \mathscr{F}^{\prime}(J) \mathscr{F}^{\prime}\left(J^{*}\right) J J^{*}}{a_{0}^{2} g_{o}^{2} \Lambda}\left(\sqrt{J}+\sqrt{J^{*}}\right) \tau_{1}^{2} \tau_{1}^{* 2} \zeta .
\end{gathered}
$$

where we should use

$$
\rho+p=-\left(\mathscr{F}^{\prime}(J) \tau_{1}^{2} J+\mathscr{F}^{\prime}\left(J^{*}\right) \tau_{1}^{* 2} J^{*}\right)
$$

Using the explicit expression for $\tau_{1}=\tau_{0} e^{\alpha t} \approx \tau_{0} e^{i \sqrt{J} t}$ and representing $\alpha=x / 2+i y / 2$ one can write for any $A$

$$
\begin{aligned}
A \tau_{1}^{2}+A^{*} \tau_{1}^{* 2} & =2 A_{0} e^{x t} \cos (y t+\varphi) \\
-i\left(A \tau_{1}^{2}+A^{*} \tau_{1}^{* 2}\right) & =2 A_{0} e^{x t} \sin (y t+\varphi)
\end{aligned}
$$

Introducing $\chi=i \frac{R}{g_{0}^{2} \Lambda} e^{x t} \zeta$ the equations of interest can be written as

$$
\begin{aligned}
& \cos (y t+r) \ddot{\chi}+2\left(\sqrt{x^{2}+y^{2}} \sin \left(y t+r+\varphi_{b}\right)-x \cos (y t+r)\right) \dot{\chi}+ \\
& +\left(\cos (y t+r)\left(6 h_{0} \sqrt{x^{2}+y^{2}} e^{x t} \sin \left(y t-\varphi_{b}\right)+\frac{k^{2}}{a_{0}^{2}}+x^{2}\right)-\right. \\
& \left.-2 x \sqrt{x^{2}+y^{2}} \sin \left(y t+r+\varphi_{b}\right)\right) \chi=-2 y \varepsilon \\
& \quad \cos (y t+r) \ddot{\varepsilon}+2 \sqrt{x^{2}+y^{2}} \sin \left(y t+r-\varphi_{b}\right) \dot{\varepsilon}+ \\
& +3 H_{0} \sqrt{\left(\frac{k^{2}}{3 a_{0}^{2} H_{0}}-x\right)^{2}+y^{2}} \cos \left(y t+r-\varphi_{c}\right) \varepsilon=\frac{2 k^{2} y}{a_{0}^{2}} \chi
\end{aligned}
$$


where all the constant coefficients are real, $\varepsilon$ and $\chi$ are real, $x$ is expected to be negative, $R>0, r$ depends on $\mathscr{F}^{\prime}(J)$, and

$$
\varphi_{b}=\arcsin \frac{x}{\sqrt{x^{2}+y^{2}}}, \varphi_{c}=\arcsin \frac{y}{\sqrt{\left(\frac{k^{2}}{3 a_{0}^{2} H_{0}}-x\right)^{2}+y^{2}}} .
$$

The most alarming points of the evolution are $y t+r=\frac{\pi}{2}+n \pi$ where the coefficients in front of second derivatives become zero. Numeric integration may hit problems at these points if the precision is not very high. In the neighborhood of these points one has

$$
\begin{gathered}
t \ddot{\chi}-2 \dot{\chi}+2 x \chi=2 \varepsilon \\
t \ddot{\varepsilon}-2 \dot{\varepsilon}-3 H_{0} \varepsilon=\frac{2 k^{2}}{a_{0}^{2}} \chi .
\end{gathered}
$$

For negative $x$ the solution for $\varepsilon$ around $t=0$ is $\varepsilon=\varepsilon_{0}+\varepsilon_{1} t+\ldots$ meaning that these points are not singular for the above system of equations.

A typical behavior for the function $\varepsilon$ is dumped oscillations. Such a behavior does not depend on the wavenumber meaning that perturbations with complex conjugate scalar fields do vanish. This is different from usual models with real scalar fields where different regimes exist and most likely growing modes are present.

This analysis can be easily extended to many complex roots and the main result, absence of growing modes at large times will remain. Indeed, the background solution is determined with the component having largest $|x|$. Thus the background quantities can be considered approximately the same. New equations (in total we have $N$ equations if we have $N$ roots) can also be accounted numerically and one can demonstrate that the main effect of absence of the growing perturbations in $\varepsilon$ does remain. The best way to see this, however, is to go to the exactly solvable system in which the analysis is more transparent. The detailed analysis of this question is to be found in the forthcoming paper [16].

\section{COUPLING TO DILATON AND THE COSMOLOGICAL SIGNATURE OF NON-LOCAL MODELS}

It is natural to consider a more general coupling of the open string modes with the closed string excitations. The first non-trivial step is to add the dilaton to the metric. The action (1) is minimally modified as follows

$$
S=\int d^{4} x \sqrt{-g}\left(\frac{e^{-\Phi}}{16 \pi G_{N}}\left(R+\left(\partial_{\mu} \Phi\right)^{2}-U(\Phi)\right)+\frac{1}{g_{o}^{2}}\left(e^{-\Phi / 2}\left(-\frac{1}{2} \partial_{\mu} \tilde{T} \partial^{\mu} \tilde{T}+\frac{1}{2} \tilde{T}^{2}\right)-\widetilde{e^{-\Phi / 2}} v(\bar{T})\right)-\Lambda^{\prime}\right) .
$$

Such a modified model was analyzed in details in [5]. Here $\Phi$ is the dilaton field and $U(\Phi)$ is the dilaton potential. The main point is that on one hand the dilaton field is dynamical while on the other hand the restrictions on the rate of change of the Newtonian constant are very narrow (for example, from the Solar system measurements). The latter circumstance allows to use a series expansion for the dilaton field around some constant value. Effectively this means that equations derived in previous Sections get modified with small corrections. However, the presence of the dynamical dilaton field dictates more restrictions on solutions and modifies the possible vacuum expectation values for the field $T$ as well. This in turn alters the spectrum of roots $J_{i}$ making the system significantly different from the the just tachyon dynamics.

There is one exceptional case for the above system when the tachyon potential has a minimum with both first and second derivatives vanishing. Physically phis means that the potential is flat in the minimum. This corresponds to the real root $J=0$ if $v(\bar{T}) \sim \bar{T}^{4}$. In this particular case dilaton corrections render small deviations from $J=0$. These corrections become comparable to the Hubble scale. It is possible because of the smallness of the dilaton rate of change. As a consequence all the parameters in the solution turn out to be of the present cosmological scales. The resulting behavior are oscillations with a period of order of $1 \mathrm{Gyr}$ in the state parameter $w$ which is detectable. Moreover, there are indications of oscillations of this kind in the literature [15].

It is the situation which one cannot easily achieve having just an open string sector while normally all the string quantities are of the order of $\alpha^{\prime}$. 


\section{SUMMARY AND OUTLOOK}

To summarize we have put in line the appearance of non-local models from SFT, construction of asymptotic solutions in such models, analysis of perturbations and a possibility to extract observationally detectable effects.

Indeed, models coming out of the UV-complete theory are interesting because they are expected to be free of pathologies. Appearance of non-localities is natural in SFT while their impact on cosmological models is a novel effect. Solutions in the non-local models in general are not constructed yet. There are numeric attempts while analytic solutions are known only in the linear models.

The example of perturbations with complex roots carried out in this note demonstrates that linear perturbations can be confined thus not destroying the system. It is not evident from the very beginning and supports the claim that the SFT based models are stable. The case of complex $J_{i}$ has never been studied in general and deserves deeper investigation. This problem and other technical questions related to the equations of the present paper will be addressed in the forthcoming publications.

Another interesting question is the study of models which possess exact solutions preserving the asymptotic behavior. This would drastically simplify the analysis of the perturbations. Also generalization of the localization method to the presence of the dilaton field is a yet unexplored problem worth further investigation.

As a more ambitious problem which is of great importance is a construction of the formalism analogous to presented in this paper for a model with self-interacting non-local scalar field. Such models play important role in the SFT. For instance, rolling tachyon dynamics is governed by action (3) with a polynomial potential of fourth degree. However, even background solutions are not very well understood because there is no general analytic way of solving non-local non-linear equations. On the other hand it follows from the present analysis that passing to a local system with many fields is vital for the construction of perturbation equations.

Looking a step further it is interesting to consider perturbations in other non-local models coming from the SFT. For instance, models where open and closed string modes are non-minimally coupled may be of interest in cosmology. An example of the classical solution is presented in [5]. Furthermore it should be possible to extend the formalism presented in this paper to other models involving non-localities like modified gravity setups.

\section{ACKNOWLEDGMENTS}

The author would like to thank the organizers of the Invisible Universe 2009 meeting for the opportunity to present the work and for creating the very stimulating environment for scientific discussions. The author is grateful to I.Ya. Aref'eva and S.Yu. Vernov for the collaboration on papers led to this presentation and to F. Bezrukov, B. Craps, B. Dragovich, G. Dvali, and V. Mukhanov for useful comments and stimulating discussions. This work is supported in part by RFBR grant 08-01-00798, state contract of Russian Federal Agency for Science and Innovations 02.740.11.5057, the Belgian Federal Science Policy Office through the Interuniversity Attraction Poles IAP VI/11, the

European Commission FP6 RTN programme MRTN-CT-2004-005104 and by FWO-Vlaanderen through the project G.0428.06.

\section{REFERENCES}

1. K. Ohmori, [hep-th/0102085];

I.Ya. Aref'eva, D.M. Belov, A.A. Giryavets, A.S. Koshelev and P.B. Medvedev, [hep-th/0111208];

W. Taylor, [hep-th/0301094].

2. L. Brekke, P.G.O. Freund, M. Olson, E. Witten, Nucl. Phys. B302 (1988) 365;

P.H. Frampton, Ya. Okada, Phys. Rev. D37 (1988) 3077;

V.S. Vladimirov, I.V. Volovich, E.I. Zelenov, "p-adic Analysis and Mathematical Physics”, WSP, Singapore, 1994;

B. Dragovich, A. Yu. Khrennikov, S. V. Kozyrev, I. V. Volovich, Anal. Appl. 1 (2009) 1, [arXiv:0904.4205].

3. I.Ya. Aref'eva, AIP Conf. Proc. 826 (2006) 301, [astro-ph/0410443];

I.Ya. Aref'eva, AIP Conf. Proc. 957 (2007) 297, [arXiv:0710.3017].

4. A.S. Koshelev, JHEP 0704 (2007) 029, [hep-th/0701103].

5. I.Ya. Aref'eva and A.S. Koshelev, JHEP 0809 (2008) 068, [arXiv:0804.3570].

6. A.S. Koshelev and S.Yu. Vernov, [arXiv:0903.5176].

7. M. Ostrogradski, Mem. Ac. St. Petersburg, VI 4 (1850) 385.

8. S.J. Perlmutter et al., Astrophys. J. 517 (1999) 565, [astro-ph/9812133];

A. Riess et al., Astron. J. 116 (1998) 1009, [astro-ph/9805201]; 
A. Riess et al., Astrophys. J. 607 (2004) 665, [astro-ph/0402512];

R.A. Knop et al., Astrophys. J. 598 (2003) 102, [astro-ph/0309368];

M. Tegmark et al., Astrophys. J. 606 (2004) 702, [astro-ph/0310723];

D.N. Spergel et al., Astrophys. J. Suppl. 148 (2003) 175, [astro-ph/0302209]

P. Astier et al., Astron. Astrophys.447 (2006) 31, [astro-ph/0510447]

W.M. Wood-Vasey et al., Astrophys. J. 666 (2007) 694, [astro-ph/0701041]

9. E. Komatsu, Astrophys. J. Suppl. 180 (2009) 330, [arXiv:0803.0547];

M. Kilbinger et al., [arXiv:0810.5129].

10. T. Padmanabhan, Phys. Rep 380 (2003) 235, [hep-th/0212290];

P. Frampton, [astro-ph/0409166];

E. J. Copeland, M. Sami, Sh. Tsujikawa, [hep-th/0603057];

A. Albrecht, et al., [astro-ph/0609591].

11. L.V. Joukovskaya, Phys. Rev. D76 (2007) 105007, [arXiv:0707.1545];

L.V. Joukovskaya, [arXiv:0807.2065].

12. J.M. Bardeen, Phys. Rev. D22 (1980) 1882.

13. V.F. Mukhanov, H.A. Feldman, and R.H. Brandenberger, Phys. Rep. 215 (1992) 203;

V.F. Mukhanov, "Physical Foundations of Cosmology", Cambridge University Press, 2005.

14. J. Hwang and H. Noh, Class. Quant. Grav. 19 (2002) 527, [astro-ph/0103244].

15. A.I. Ryabinkov, A.D. Kaminker, D.A. Varshalovich, Mon. Not. Roy. Astron. Soc. 376 (2007) 1838, [astro-ph/0703277].

16. F. Galli and A.S. Koshelev, to appear. 